\title{
Analysis of the possibility of using high-speed pneumatic directional valves to regulate the flow of compressed air
}

\author{
Jakub Takosoglu, ${ }^{1, *}$ \\ ${ }^{1}$ Faculty of Mechatronics and Mechanical Engineering, Department of Manufacturing Engineering and Metrology, Kielce University of \\ Technology, Aleja Tysiaclecia Panstwa Polskiego 7, 25-314 Kielce, Poland
}

\begin{abstract}
Most often, proportional flow valves or flow servovalves are used for position control of pneumatic drives. In case of this type of valves, flow characteristic of the valve represents a dependence of flow rate upon analogue control signal (voltage or current). The paper presents experimental studies of two high-speed pneumatic 3/2 directional valves (3-way 2-position) in order to determine the flow characteristics and flow parameters. One has analysed the option to use the directional valves to control the flow rate of compressed air using frequency-based signals.
\end{abstract}

\section{Introduction}

Compressed air is an important energy carrier in the industry. Air is open for general use, it is safe and clean during operation; compressed air is resistant to temperature fluctuations. It can be transported to significant distances and it is used as a working medium during decompression. Due to benefits of the working medium, pneumatic drives are commonly used in every sector of the industry (medicine, transport, aviation, automotive, light and heavy industries, mining, metallurgy) as well as in our everyday lives (pneumatic tools, tyre repair shops, odontology) [1]. Point to point control is most often used in the industrial applications. This manner of positioning consists in movement of a cylinder piston from the initial position A to the final position $B$ within shortest possible time. There are several methods for positioning pneumatic drives, however, even though these are very accurate and repeatable methods, they are limited to a few predetermined positions or are limited due to application options. The most promising and showing the most potential is the chocking method in case of which sophisticated control methods are designed, e.g. with an algorithm using artificial intelligence methods [2]. Civilization and technological development enforces changes in the pneumatic systems towards more simplicity of installation and operation with simultaneous improvement of precision in pneumatic devices application. This necessitates application of electronics and pneumatic proportional technique, which continuously amplifies and converts weak input signal into strong output signal in terms of flow rate (flow valves) or pressure (pressure valves). Proportional valves represent a link connecting pneumatic systems with electronic control system. The proportional technique within fluid-based systems has been introduced for the first time in hydraulic systems, and only in the Eighties it has been implemented in pneumatic systems. Proportional valves are used first and foremost in fast-acting control systems in the production processes in order to control:

- working medium flow direction,

- working medium flow rate,

- working medium pressure (power or torque).

They can also be used in the industry to:

- control the power of pneumatic cylinders,

- control the speed of pneumatic cylinders,

- control the speed of pneumatic turbines,

- control the speed of pneumatic tools,

- control the pressure or flow rate in paint spraying guns,

- batch and transport powder materials,

- pneumatic positioning,

- active vibrations dampening.

Pneumatic proportional valves and servovalves are structurally and technologically complicated elements, therefore they are expensive. The purpose of the paper is the option to use the high-speed directional valves [3-6] to control the flow rate [7] of compressed air using frequency-based signals. Fig. 1 presents a diagram with $2 / 2$ vales, and fig. 2 a diagram with $3 / 2$ valves.

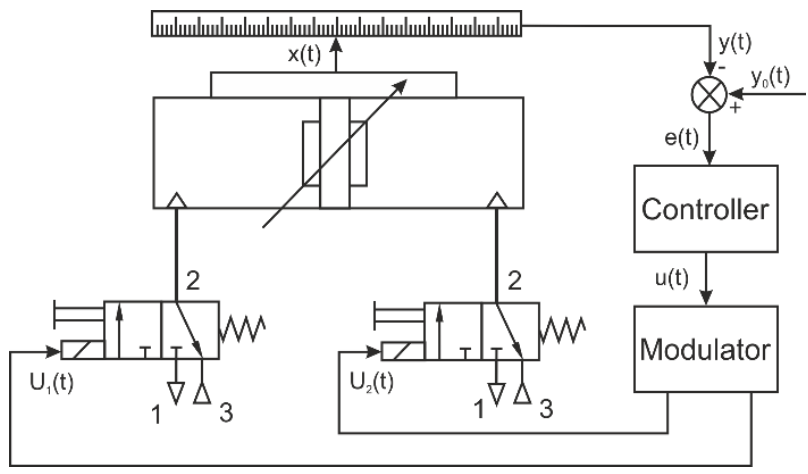

Fig. 1. Diagram of a drive with choking control with two $3 / 2$ directional valves. 
Changes of flow into a cylinder chambers are achieved by suitable modulation of control voltage of valve coils. Regulator operating in the negative feedback loop in relation to the position signal generates a control signal to a modulator.

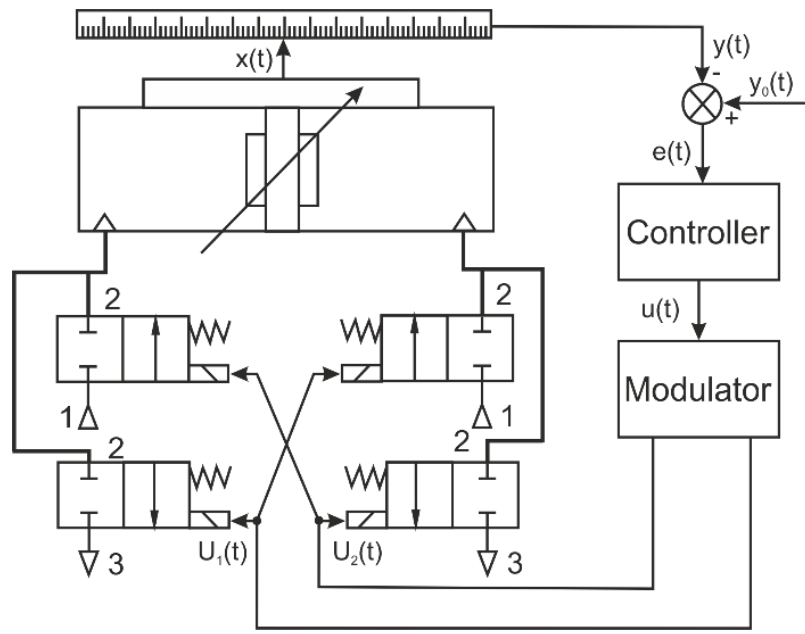

Fig. 2. Diagram of a drive with choking control with four $2 / 2$ directional valves.

On the diagram presented in fig. 1 , it is necessary to use 2 $3 / 2$ directional valves and on diagram presented in fig. 2 $42 / 2$ directional valves. One selected the less complicated system and decided to study two types of high-speed $3 / 2$ directional valves such as bullet valves, manufactured by MAC. Table 1 presents parameters of the valve BV309ACD1-00-BGEA-CTA, and table 2 presents parameter of the valve BV310A-CD1-00-BHEA-CTA.

Table 1. Parameters of the valve BV309A-CD1-00-BGEACTA.

\begin{tabular}{|l|c|}
\hline \multicolumn{1}{|c|}{ Parameter } & Value \\
\hline Symbol & $\begin{array}{c}\text { BV309A-CD1-00-BGEA- } \\
\text { CTA }\end{array}$ \\
\hline Nominal size & $8.6 \mathrm{~mm}$ \\
\hline Valve function & $3 / 2$-way, normally closed \\
\hline Pressure range & Vacuum to $0.83 \mathrm{MPa}$ \\
\hline Temperature range & $-18^{\circ} \mathrm{C}$ to $+50^{\circ} \mathrm{C}$ \\
\hline Flow factor & Up to $0.06 \mathrm{Cv}$ \\
\hline Filtration & $40 \mu \mathrm{m}$ \\
\hline Voltage & 24 VDC \\
\hline
\end{tabular}

Table 2. Parameters of the valve BV310A-CD1-00-BHEACTA.

\begin{tabular}{|l|c|}
\hline \multicolumn{1}{|c|}{ Parameter } & Value \\
\hline Symbol & $\begin{array}{c}\text { BV310A-CD1-00-BHEA- } \\
\text { CTA }\end{array}$ \\
\hline Nominal size & $9.4 \mathrm{~mm}$ \\
\hline Valve function & $3 / 2$-way, normally closed \\
\hline Pressure range & Vacuum to $0.83 \mathrm{MPa}$ \\
\hline Temperature range & $-18^{\circ} \mathrm{C}$ to $+50^{\circ} \mathrm{C}$ \\
\hline Flow factor & Up to $0.09 \mathrm{Cv}$ \\
\hline Filtration & $40 \mu \mathrm{m}$ \\
\hline Voltage & $24 \mathrm{VDC}$ \\
\hline
\end{tabular}

General view of the valves is presented in Fig. $3 \mathrm{a}$ and $3 \mathrm{~b}$ respectively, however Fig. $3 \mathrm{c}$ present pneumatic symbols of the valves. a)

c)

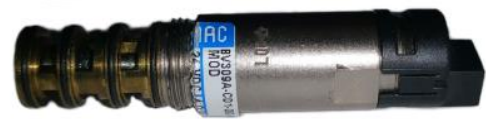

b)
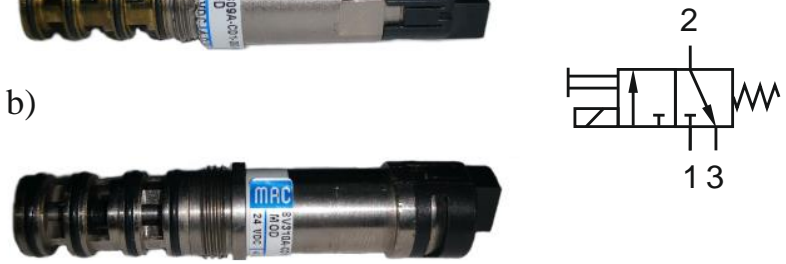

13

Fig. 3. General view of directional valve : a) BV309A-CD1-00BGEA-CTA, b) BV310A-CD1-00-BHEA-CTA, c) pneumatic symbol.

Fig. 4 presents a simplified diagram of the internal structure of the high-speed directional valve.

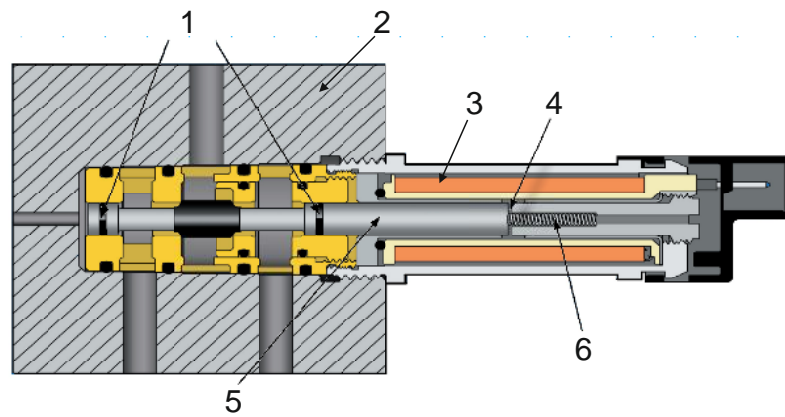

Fig. 4. Internal structure of high-speed directional valve 1 -seals, 2 - housing, 3 - electromagnetic transducer, 4 - overstroke, 5 - poppet, 6 - return spring.

\section{Test stand}

All four high-speed 3/2 directional valves were installed on a special connection panel (Fig. 5). The panel allows for connecting two double-acting cylinders and performing studies related to cylinders positioning using high-speed directional valves. The panel with valves functions as valve terminal [8].

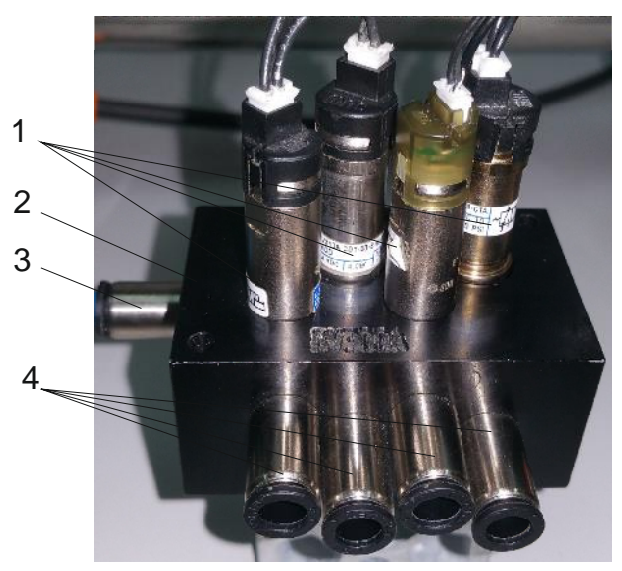

Fig. 5. General view of the valves on the panel : 1 - valves, 2 - panel, 3 - air supply, 4 - outlet way (to cylinders).

The studies were performed using only two valves at a time. Computer system using Rapid Control Prototyping (RPC) technology was used to generate the control signals. The system operates based on Matlab xPC 
Target software, PCs and card with DA/AD converters. DA converters generate voltage of maximum value 10 $\mathrm{VDC}$, however $\mathrm{AD}$ converters generate voltage of maximum value 5 VDC. Both types of converters are lowcurrent devices. Due to necessity to control the valves using voltage of value $24 \mathrm{VDC}$ and power $4.0 \mathrm{~W}$, an electronic system was made, diagram of which is presented in Fig. 6.

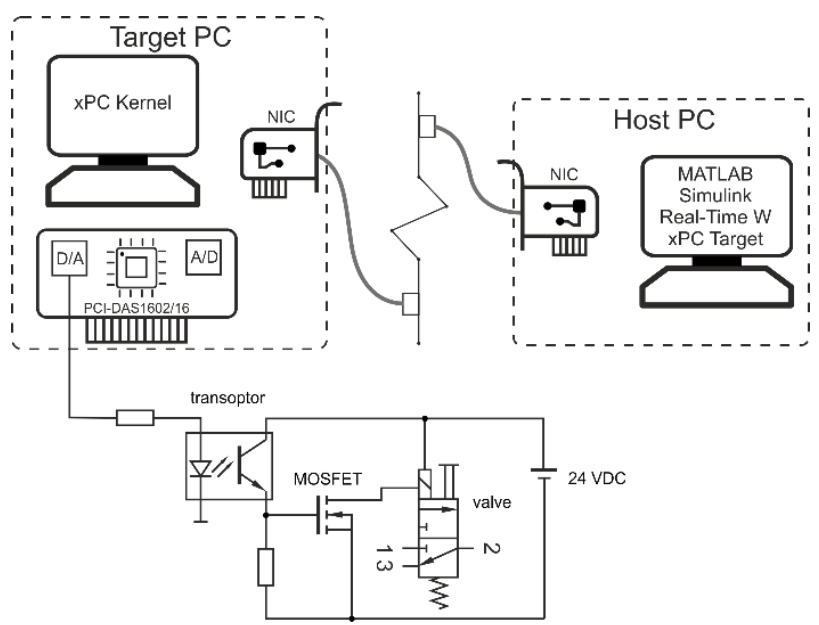

Fig. 6. Electronic system diagram.

Diagram of the testing stand for determining flow characteristics of directional valves pilots is presented in Fig. 7, and the general view of the stand is presented in Fig. 8.

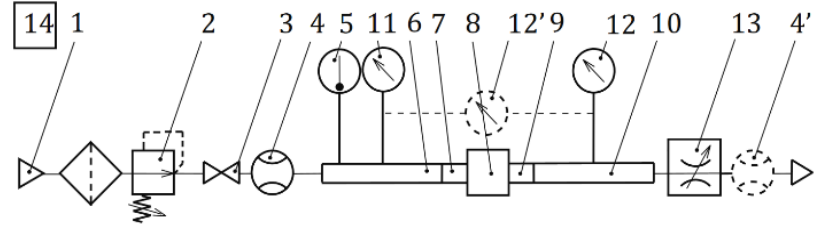

Fig. 7. Diagram of the stand for determining flow characteristics: 1 - compressed gas source and filter, 2 adjustable pressure regulator, 3 - shut-off valve, 4 - flow-rate measuring device, 5 - temperature-measuring instrument, 6 upstream pressure-measuring tube, 7 - upstream transition connector, 8 - component under test, 9 - downstream transition connection, 10 - downstream pressure-measuring tube, 11 upstream pressure gauge or transducer, 12 - downstream pressure gauge or transducer, 13 - flow control valve, 14 barometer.

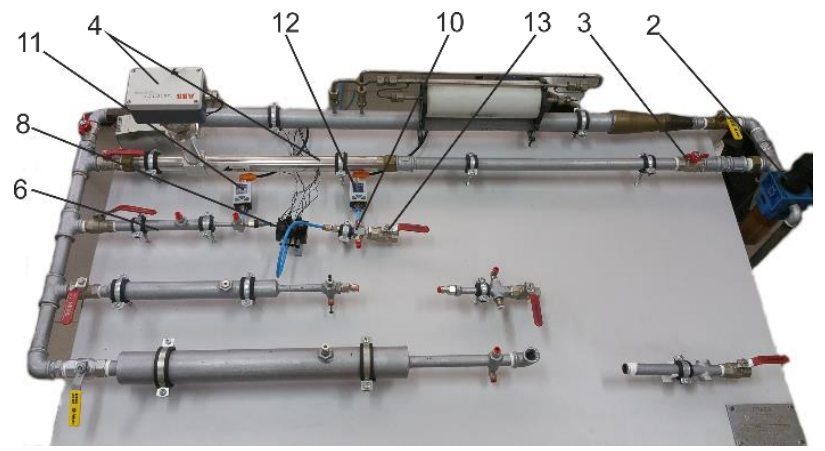

Fig. 8. General view of the stand.
Method of performing the studies on the stand, description of the stand and description of the flow characteristics are presented in detail in the paper [7, 8].

\section{Results of the experimental studies}

At the first stage of the studies, one determined the maximum value of flow rate through the valves in the function of the supply pressure (Fig. 9 and 10). In the initial phase of the pressure increase from 0.0 to $0.05 \mathrm{MPa}$, a very small volume of compressed air flows by valve, below the measuring range of the flowmeter. Above pressure $0.05 \mathrm{MPa}$ there is a more intensive flow of compressed air (at $0.1 \mathrm{MPa}: 5 \mathrm{Nl} / \mathrm{min}$ and $7 \mathrm{Nl} / \mathrm{min}$ respectively for the BV310A-CD1-00-BHEA-CTA valve and BV309A-CD1-00-BGEA-CTA valve).

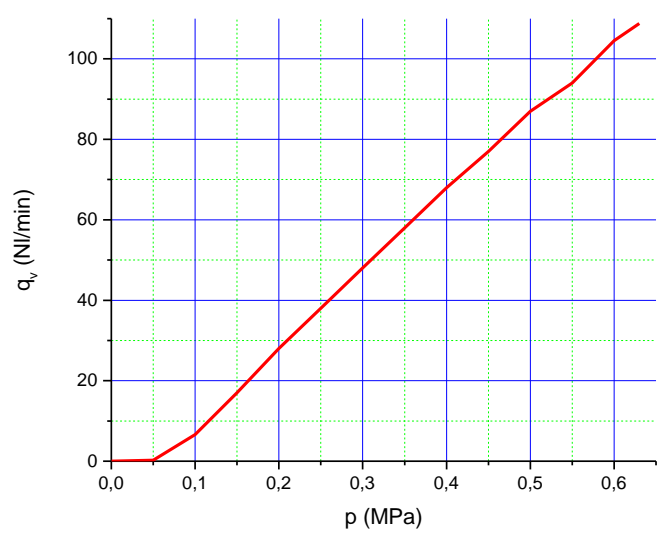

Fig. 9. Characteristic $q_{v}=f(p)$ of the valve BV309A-CD1-00BGEA-CTA.

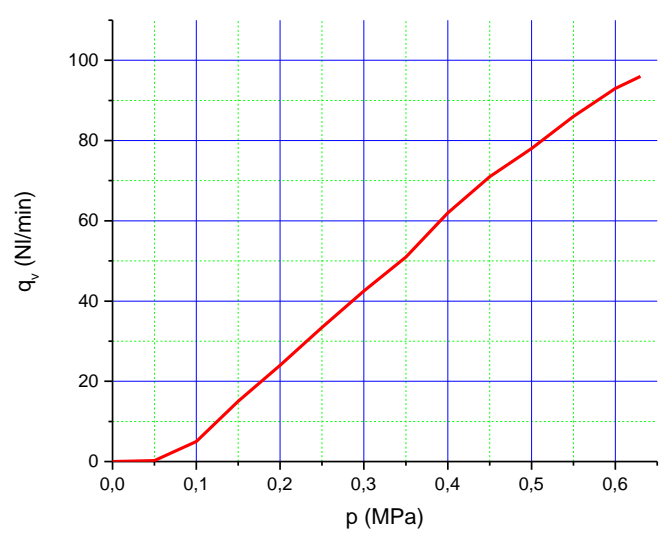

Fig. 10. Characteristic $q_{v}=f(p)$ of the valve BV310A-CD1-00BHEA-CTA.

Next, in the system presented in Fig. 6, one supplied the frequency-based signal to the directional valve coils within the range of $10-400 \mathrm{~Hz}$ and then measured the flow rate values (Fig. 11 and 12). 


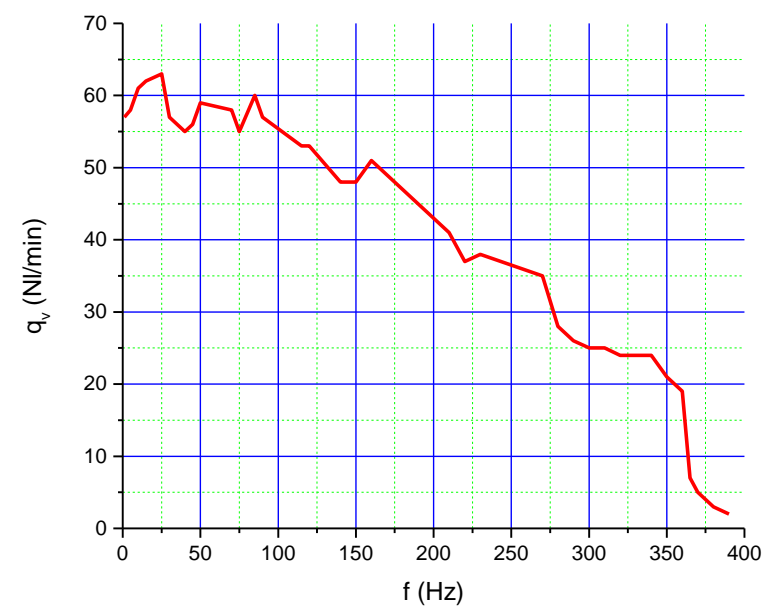

Fig. 11. Characteristic $q_{v}=f(f)$ of the valve BV309A-CD1-00BGEA-CTA.

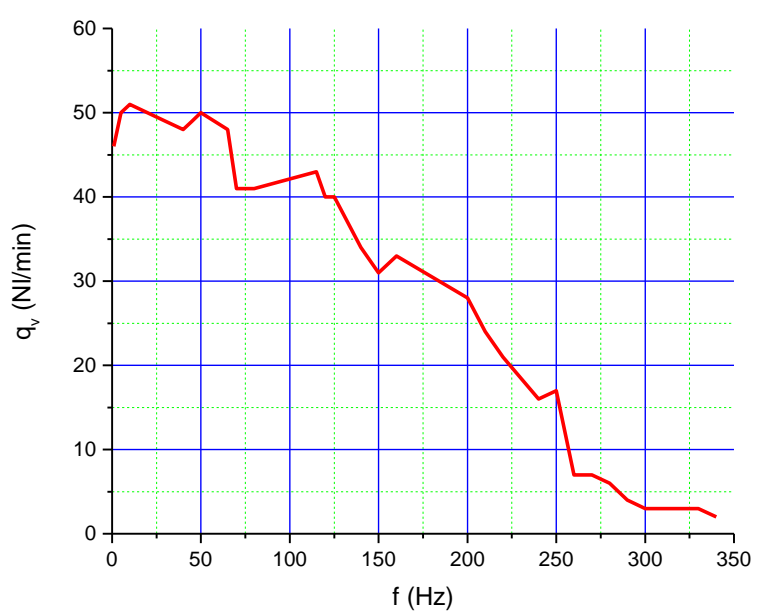

Fig. 12. Characteristic $q_{v}=f(f)$ of the valve BV310A-CD1-00BHEA-CTA.

\section{Summary}

The paper presents experimental studies of two types of high-speed pneumatic 3/2 directional valves (3-way 2position) in order to determine the flow characteristics. One has analysed the option to use the directional valves to control the flow rate of compressed air using frequency-based signals. Maximum value of flow rate is $q_{v}=107.2 \mathrm{Nl} / \mathrm{min}$ for the valve BV309A-CD1-00-BGEACTA, and $q_{v}=96.1 \mathrm{Nl} / \mathrm{min}$ for the valve BV310A-CD1-00BHEA-CTA. Together with the increase in the control signal frequency, flow rate drops to the limit value of $q_{v}=2.01 / \mathrm{min}$ for the valve BV309A-CD1-00-BGEA-CTA, corresponding to frequency of $390 \mathrm{~Hz}$ resulting from the option to switch the valve (390 times per second). In case of the valve BV310A-CD1-00-BHEA-CTA, the limit value of flow rate is $q_{v}=2.01 / \mathrm{min}$ at frequency $340 \mathrm{~Hz}$ ( 340 times per second). Due to the obtained characteristics and extremely long life of the valves exceeding billion cycles, the valves can be successfully used to control the compressed air flow rate. Moreover, the obtained studies results are the basis for the works concerning positional control of double-acting cylinder, as presented in Fig. 2.

\section{References}

1. J.E. Takosoglu, R.F. Dindorf, P.A. Laski, Int. J. Adv. Manuf. Technol. 40, 3-4(2009)

2. J.E. Takosoglu, P.A. Laski, S. Blasiak, Proc. Inst. Mech. Eng. Part I J. Syst. Control Eng. 226, 10 (2012)

3. S. Hodgson, M.Q. Le, M. Tavakoli, M.T. Pham, Mechatronics. 22, 1 (2011)

4. Q. Minh Le, M.T. Pham, M. Tavakoli, R. Moreau, J.P. Simon, T. Redarce, IEEE Trans. Control Syst. Technol. 21, 4 (2013)

5. F. Najafi, M. Fathi, M. Saadat, Int. J. Adv. Manuf. Technol. 42, 7-8 (2009)

6. B. Najjari, S.M. Barakati, A. Mohammadi, M.J. Futohi, M. Bostanian, ISA Trans. 53, 2 (2014)

7. S. Blasiak, J.E. Takosoglu, P.A. Laski, D.S. Pietrala, J. Zwierzchowski, G. Bracha, et al., (2016)

8. J.E. Takosoglu, P.A. Laski, S. Blasiak, (Brno Univ. Technol., Inst. Solid Mechan. Mechatron. \& Biomechan., 2014)

9. J.E. Takosoglu, (Techn. Univ. Liberec, 2016)

10. S. Blasiak, J.E. Takosoglu, P.A. Laski, (Brno Univ. Technol., Inst. Solid Mechan. Mechatron. \& Biomechan., 2014) 